\title{
Bioinformatics analysis of dysregulated microRNAs in the nipple discharge of patients with breast cancer
}

\author{
KAI ZHANG, YA-WEN WANG and RONG MA \\ Department of Breast Surgery, Qilu Hospital of Shandong University, Jinan, Shandong 250012, P.R. China
}

Received October 18, 2015; Accepted January 13, 2017

DOI: $10.3892 / 01.2017 .5801$

\begin{abstract}
MicroRNAs (miRNAs/miRs) have been reported to be associated with the tumorigenesis and progression of various types of human cancer; however, the underlying mechanisms of this association remain unclear. The aim of the present study was to explore the potential functions of miRNAs in the development of breast cancer using bioinformatics analysis, based on the miRNA expression profile in nipple discharge. A previous study demonstrated the upregulation of miR-3646 and miR-4484, and the downregulation of miR-4732-5p in the nipple discharge of patients with breast cancer, compared with patients with benign breast lesions. In the present study, the target genes of miR-3646, -4484 and -4732-5p were predicted using TargetScan and the MicroRNA Target Prediction and Functional Study Database. The predicted target genes were further analyzed by Gene Ontology term enrichment analysis, Kyoto Encyclopedia of Genes and Genomes pathway enrichment analysis and protein-protein interaction analysis. Numerous carcinoma-associated genes, including ADIPOQ, CPEB1, DNAJB4, EIF4E, APP and BCLAF1, were revealed to be putative targets of miR-3646, -4484 and $-4732-5 p$. Bioinformatics analysis associated miR-3646 with the Rap1 and TGF- $\beta$ signaling pathways, miR-4484 with the ErbB, estrogen and focal adhesion signaling pathways, and miR-4732-5p with the proteoglycan signaling pathway. Notably, protein-protein interaction analysis identified that numerous predicted targets of these miRNAs were associated with one other. In addition, the target genes of the miRNAs were identified to be under the regulation of a number of transcription factors (TFs). The predicted target genes of miR-3646, -4484 and -4732-5p were identified to serve a role in cancer-associated signaling pathways and TF-mRNA networks, indicating that they serve a role in breast carcinogenesis and progression. These results
\end{abstract}

Correspondence to: Professor Rong Ma, Department of Breast Surgery, Qilu Hospital of Shandong University, 107 West Wenhua Road, Jinan, Shandong 250012, P.R. China

E-mail: marongw2000@163.com

Key words: breast cancer, nipple discharge, bioinformatics analysis, target genes, pathway, transcription factor-mRNA network provide a comprehensive view of the functions and molecular mechanisms of miR-3646,-4484 and -4732-5p, and will aid in future studies.

\section{Introduction}

Breast cancer is one of the most common types of cancer diagnosed in women worldwide (1), and nipple discharge is one of the most common symptoms in women presenting with breast complaints (2). Intraductal papilloma, a benign tumor, is the most common cause of abnormal nipple discharge (3); however, $\sim 5-15 \%$ of cases are associated with a malignant lesion $(4,5)$. There are no clear guidelines on what differentiates benign with malignant etiology based on clinical and radiographic assessments (6), and $>20 \%$ of patient with cancer have no obvious clinical or radiological evidence of breast carcinoma (7). The use of nipple discharge cytology for diagnosis is currently limited due to its low sensitivity of $7 \%$ (8); therefore, the identification of novel biomarkers in nipple discharge to detect breast cancer at an early stage is essential for improving the treatment and survival of patients.

MicroRNA (miRNA/miR) is a class of non-coding small RNA that can induce mRNA degradation and/or translational repression, through binding to the 3 ' untranslated region (UTR) of mRNAs and thereby negatively regulating the transcription of target genes (9). It has been demonstrated that miRNAs are associated with numerous essential biological processes, including proliferation, apoptosis, development and differentiation, in addition to human cancer initiation and progression $(10,11)$. Previous studies have demonstrated that the deregulation of miRNAs in human cancer is tissueand/or time-specific, highlighting their potential roles as biomarkers $(12,13)$. For example, let-7 was identified as a diagnostic biomarker for certain lung cancer subtypes, in addition to a prognostic marker for disease progression and response to treatment (14-16). Cui et al (17) reported that miR-21 and miR-106a in gastric secretions are potential diagnostic biomarkers for gastric cancer. Improved understanding of the molecular mechanisms by which miRNAs function in breast cancer will be beneficial for the development of novel approaches for the early detection and monitoring of breast cancer. A previous study identified a panel of miRNAs, including miR-3646, -4484 and -4732-5p, in nipple discharge as potential diagnostic biomarkers for breast cancer (18). In 
the present study, the potential functions of miR-3646, -4484 and $-4732-5 \mathrm{p}$, and the miRNA-associated pathways in breast cancer, were analyzed.

\section{Materials and methods}

Target gene prediction. The target gene prediction databases Targetscan (version 6.2; http://www.targetscan.org) and MicroRNA Target Prediction and Functional Study Database (miRDB; http://mirdb.org/miRDB) (19) were used to identify potential targets of miR-3646, -4484 and $-4732-5 p$.

Enrichment analysis. A gene ontology (GO) term enrichment analysis (http://www.geneontology.org) (20) was performed to identify biological processes, molecular functions and cellular components associated with the target genes of miR-3646, miR-4484 and miR-4732-5p predicted by Targetscan. Kyoto Encyclopedia of Genes and Genomes (KEGG) pathway enrichment analysis (http://www.genome. $\mathrm{jp} / \mathrm{kegg}$ ) (21) was used to identify the miRNA targets associated with signaling pathways in breast cancer. $\mathrm{P}<0.05$ was considered to indicate a statistically significant difference. For better visualization, the top ten GO terms and pathways (if any) were presented. A protein-protein interaction analysis using the Search Tool for the Retrieval of Interacting Genes (version 9.1; http://string91 .embl.de/) database (22) was performed to test whether the targets were associated with each other.

Transcription factor (TF)-mRNA network construction. The potential TF-mRNA pairs were obtained from the tfbsConsSites table, which contains transcription factor binding sites conserved in the human/mouse/rat alignment (downloaded from the University of California Santa Cruz genome browser; http://genome.ucsc.edu/cgi-bin/hgTables). Binding sites located in the promoter region (from-2,000 bp to $+500 \mathrm{bp}$ of the transcription start site) of specified coding genes and with $\mathrm{Z}$ scores $>2.33$ were selected for further processing. Subsequently, Cytoscape version 3.2.0 software (http://www. cytoscape.org/) was used to visualize the TF-mRNA regulatory network.

\section{Results}

Potential target genes of miR-3646,-4484 and -4732-5p. The miRDB and Targetscan databases were used to predict the target genes of miR-3646, -4484 and -4732-5p. The numbers of target genes predicted by the databases for miR-3646, -4484 and $-4732-5 p$ were 574, 12 and 10, respectively (Fig. 1A-C). Based on the prediction databases scores and the literature, a number of the target genes were selected for further investigation (Fig. 1D). The selected target genes included adiponectin, C1Q and collagen domain containing adiponectin (ADIPOQ), A-kinase anchoring protein 12, coiled-coil domain containing (CCDC) 50, CCDC6, cytoplasmic polyadenylation element-binding protein 1 (CPEB1), DnaJ heat shock protein family (Hsp40) member B4 (DNAJB4) and eukaryotic translation initiation factor $4 \mathrm{E}$ (EIF4E) for miR-3646; amyloid precursor protein (APP), EIF4E, methionine-R-sulfoxide reductase B3, patched 2 and
TATA-box binding protein associated factor 4 for miR-4484; and ALKBH5, ankyrin repeat domain 17, BCL2-associated transcription factor 1 (BCLAF1) and protein phosphatase $1 \mathrm{E}$ for miR-4732-5p. Notably, EIF4E was identified as a mutual target of miR-3646 and miR-4484.

GO and KEGG enrichment analysis of the putative targets of $\mathrm{miR}-3646,-4484$ and-4732-5p. GO term enrichment analysis was performed for the selected putative targets of miR-3646, -4484 and -4732-5p. The results revealed that the targets were significantly involved in a number of biological, cellular and molecular processes $(\mathrm{P}<0.05$; Fig. 2). KEGG pathway enrichment analysis revealed that the miRNA targets were implicated in a number of pathways that are associated with carcinogenesis and tumor progression (Fig. 3). The miR-3646 targets $(\mathrm{P}<0.05$; Fig. 3A) were significantly implicated in ubiquitin mediated proteolysis, the thyroid hormone signaling pathway, the $\gamma$-aminobutyric acidergic and cholinergic synapses, retrograde endocannabinoid signaling, axon guidance, the Ras-related protein Rap1 signaling pathway, amphetamine addiction, oocyte meiosis and the transforming growth factor $\beta$ (TGF- $\beta$ ) signaling pathway. The miR-4484 targets $(\mathrm{P}<0.05$; Fig. 3B) were significantly implicated in epidermal growth factor receptor (ErbB) signaling, estrogen signaling, chronic myeloid leukemia, bacterial invasion of epithelial cells, insulin signaling, chemokine signaling, endocytosis, focal adhesions, the cholinergic synapse and adherens junction. For the miR-4732-5p targets $(\mathrm{P}<0.05$; Fig. $3 \mathrm{C})$ the significantly enriched pathways were proteoglycans in cancer and ubiquitin mediated proteolysis.

Protein-protein interaction analysis. Protein interaction analysis was performed to investigate the interactions of the proteins translated from the mRNA targets of miR-3646, -4484 and $-4732-5 p$. The data identified a number of proteins that have a potential association with each other (Fig. 4), suggesting that the miRNA-regulated genes may form a crosstalk network in modulating breast cancer development and progression.

TF-mRNA regulatory network construction. Following the identification of TF-mRNA pairs using the UCSC genome browser, a TF-mRNA regulatory network was constructed (Fig. 5). The data suggested that the miRNA target genes are under the regulation of numerous transcription factors, including cyclic AMP-dependent transcription factor (ATF), cyclic AMP-responsive element-binding protein 1 (CREB), CCAAT/enhancer-binding protein $\alpha(\mathrm{C} / \mathrm{EBP} \alpha)$, proto-oncogene c-Fos (c-Fos), transcription factor AP-1 (c-Jun), myc proto-oncogene protein (c-Myc), transcription factor E2F1 (E2F), transcription factor JunB and JunD (JunB and JunD), cellular tumor antigen p53 (p53), transcription factor SP1 (SP1), signal transducer and activator of transcription 3 (STAT3), and transcription factor p65 (NF- $\kappa \beta)$. For miR-4484, ZSWIM6 was the target gene regulated by the majority of TFs ( $n=28$; Fig. 5A). For miR-4732-5p, ANKRD17 was the target gene regulated by the largest number of TFs ( $n=24$; Fig. 5B), and for miR-3646, EIF4E was the target gene regulated by the largest number of TFs $(n=25$; Fig. 5C). 
A

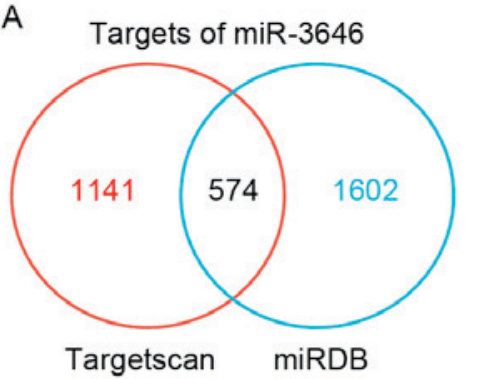

B

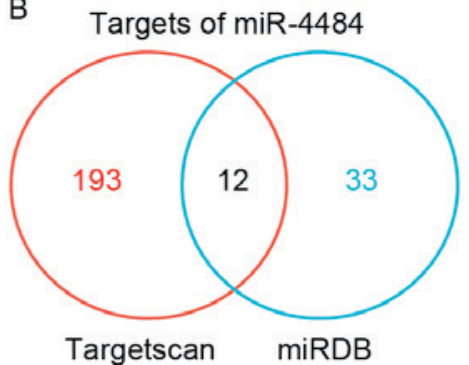

C Targets of miR-4732-5p

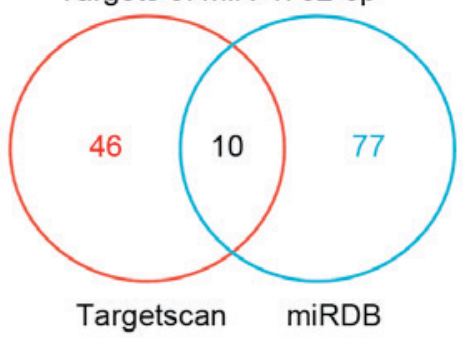

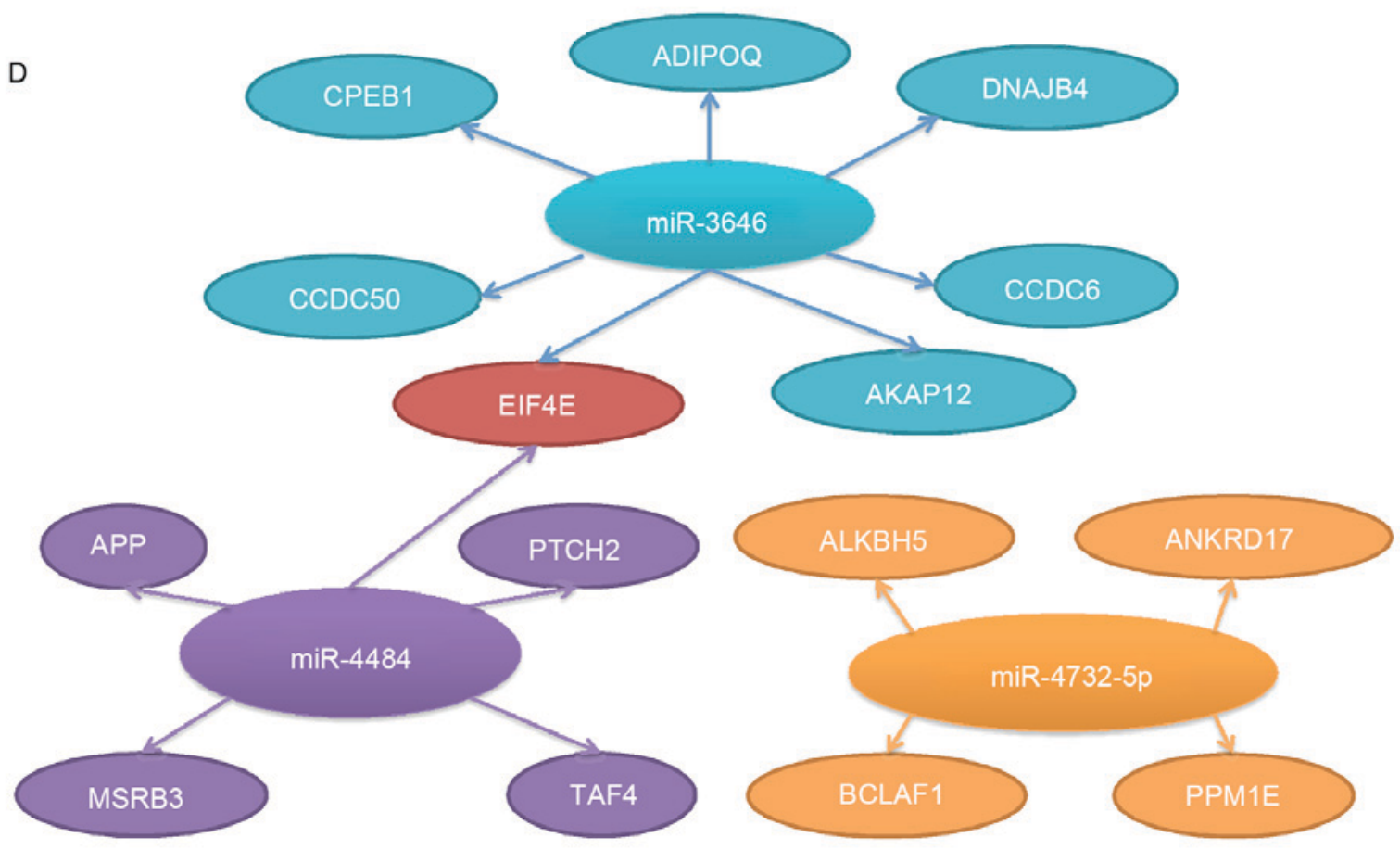

Figure 1. Predicted target genes for miR-3646, -4484 and $-4732-5 \mathrm{p}$. The number of the potential targets predicted by Targetscan and miRDB are illustrated for miR (A) -3646, (B) -4484 and (C) -4732-5p. (D) Representative predicted targets of miR-3646, $-4484,-4732-5$ p. The main criteria for selecting representative genes are based on their functions from previous cancer-associated studies, in combination with the targets scores provided by Targetscan (cumulative weighted context and score) and miRDB (Target Score). miR, microRNA; miRDB, MicroRNA Target Prediction and Functional Study Database; CPEB1, cytoplasmic polyadenylation element-binding protein 1; APP, amyloid precursor protein; ADIPOQ, C1Q and collagen domain containing adiponectin; DNAJB4, DnaJ heat shock protein family (Hsp40) member B4; CCDC, coiled-coil domain containing; AKAP12, A-kinase anchoring protein 12; EIF4E, eukaryotic translation initiation factor 4E; PTCH2, patched 2; TAF4, TATA-box binding protein associated factor 4; MSRB3, methionine-R-sulfoxide reductase B3; ANKRD17, ankyrin repeat domain 17; PPM1E, protein phosphatase 1E; BCLAF1, BCL2-associated transcription factor 1.

\section{Discussion}

Nipple discharge is one of the most common complaints for patients with breast lesions (23). Identifying biomarkers in nipple discharge that would allow breast cancers and benign breast lesions to be distinguished, which traditional radiological and cytological examinations cannot do, would be critical in improving diagnosis $(24,25)$. The expression signatures of miRNAs can be informative in cancer diagnosis (13). The dysregulated expression of miR-3646, -4484 and -4732-5p in the nipple discharge of patients with breast cancer compared with that of patients with benign breast lesions has previously been reported (26), implicating miRNAs as novel tumor biomarkers for the diagnosis of breast cancer. In the present study, bioinformatics analysis was performed to explore the potential functions and underlying mechanisms of miR-3646, -4484 and $-4732-5 p$ in breast cancer.

The results of the present study revealed that numerous genes associated with cell proliferation, apoptosis and metastasis are potential targets of miR-3646, -4484 and $-4732-5$ p. For example, DNAJB4, a predicted target of miR-3646 in the present study has been reported to be downregulated in colorectal carcinoma and correlated with cancer cell metastasis, disease progression and patient survival (27). Simões-Correia et al (28) demonstrated that the expression level of DNAJB4 was decreased in human gastric cancer and inhibited cancer cell invasion in vivo. DNAJB4 has also been identified as a tumor suppressor in lung cancer, in which it inhibits cell proliferation, anchorage-independent growth, tumorigenesis, cell motility and invasion $(29,30)$. Another target of miR-3646, ADIPOQ, has been associated with breast cancer risk (31) and cell invasion in breast cancer (32). More recently, Chen et al (33) identified ADIPOQ as one of the most downregulated genes in breast cancer in the Asian population. CPEB1, another potential target of mIR-3646, is downregulated in breast, ovarian and gastric cancers (34-36) and is associated with cell growth, senescence and migration in glioma (37,38). APP, a potential 


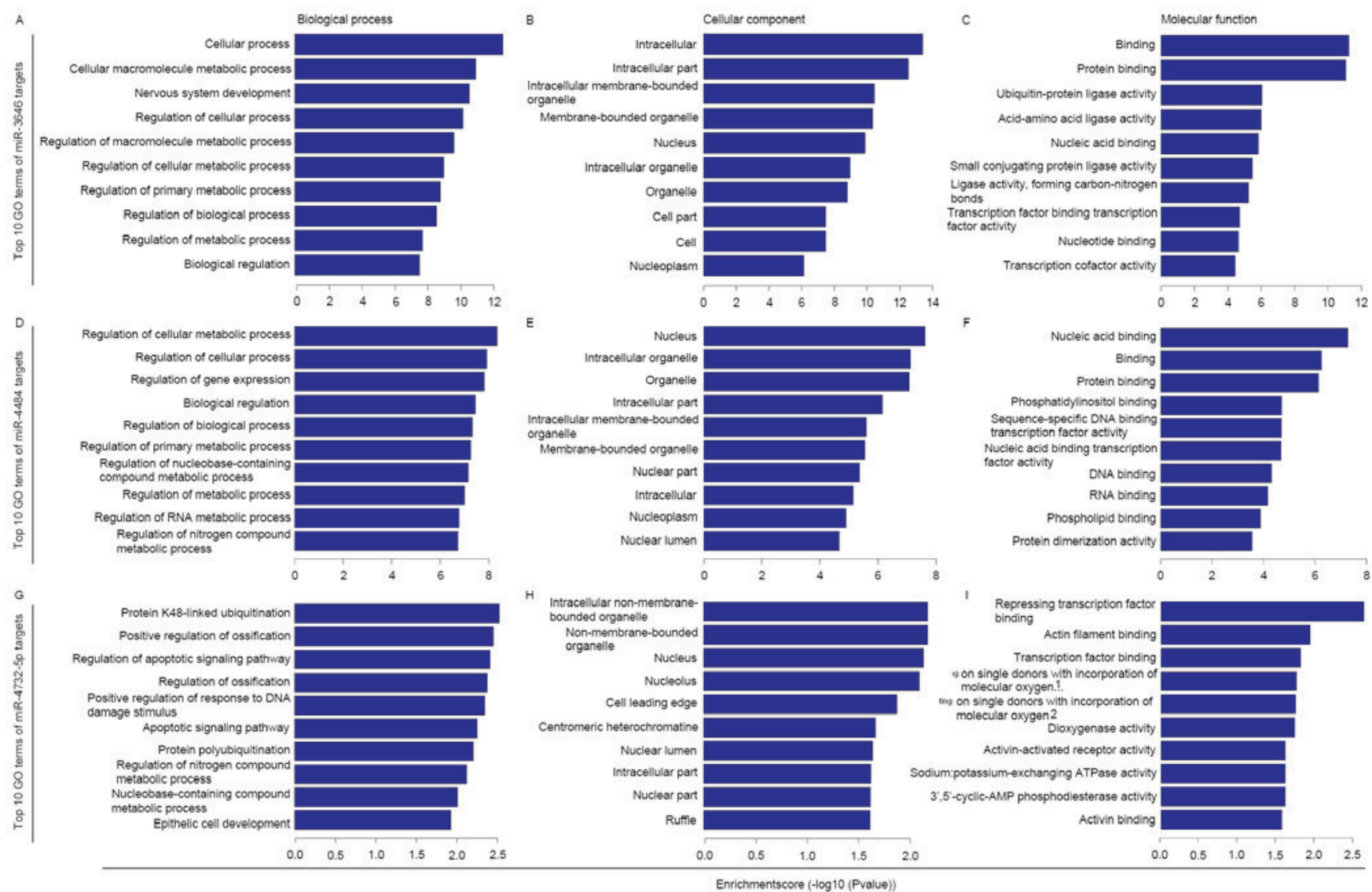

Figure 2. GO term enrichment analysis of predicted miR target genes associated with biological processes, cellular components and molecular functions. Top $10 \mathrm{GO}$ terms of miR-3646 targets for (A) biological process, (B) cellular component and (C) molecular functions. Top $10 \mathrm{GO}$ terms of miR-4484 targets for (D) biological process, (E) cellular component and (F) molecular functions. Top $10 \mathrm{GO}$ terms of miR-4732-5p targets for (G) biological process, (H) cellular component and (I) molecular functions. 'oxidoreductase activity, acting on single donors with incorporation of molecular oxygen, incorporation of two atoms of oxygen. ${ }^{2}$ oxidoreductase activity, acting on single donors with incorporation of molecular oxygen. miR, micro RNA; GO, gene ontology.

target of miR-4484, has been implicated in several human malignancies and contributes to the proliferation and migration of prostate cancer cells by modulating the expression of metalloproteinases and epithelial-mesenchymal transition-associated genes (39). The apoptosis-associated gene BCLAF1 (40), a potential target of miR-4732-5p, has been associated with tumor recurrence in esophageal squamous cell carcinoma (41).

Notably, EIF4E was identified as a shared target of miR-3646 and miR-4484. EIF4E is deregulated in a number of human malignancies, including lung, bladder, colon, breast, prostate, cervical and ovarian cancer (42). Pettersson et al (43) suggested that EIF4E silencing could reduce the invasiveness and metastatic capability of breast cancer cells. EIF4E is overexpressed in invasive breast ductal carcinoma and is associated with the occurrence and metastasis of breast cancer (44). Recently, high EIF4E expression has been correlated with an increased risk of systemic metastasis in patients with lymph node-positive breast cancer patients (45). There results indicate that miR-3646, -4484 and $-4732-5 p$ may be associated with several essential cellular processes, including cell proliferation, apoptosis and metastasis, and serve critical roles in cancer initiation and progression.

In order to investigate the underlying functional mechanisms of miR-3646, -4484 and -4732-5p KEGG pathway enrichment analysis was performed. As expected, the target genes of these miRNAs were significantly enriched in several cancer-associated signaling pathways, including those of Rap1, TGF- $\beta$, ErbB, estrogen, focal adhesion and proteoglycans. Spanjaard et al (46) demonstrated that focal adhesion size, sliding and intensity were regulated by the Rap1 signaling pathway and in turn could influence tumor metastasis. High Rap1 activity is correlated with poor differentiation and an advanced tumor stage in serous ovarian cancer (47). The Rap1 signaling pathway has also been associated with breast tumor cell migration and invasion, and breast cancer progression (48-51). TGF- $\beta$ signaling regulates diverse cellular processes, including cell proliferation, differentiation, apoptosis, plasticity and migration, and thus serves important roles in the development and progression of various kinds of diseases, including human cancer (52). TGF- $\beta$ serves a tumorigenic role, but it can also act as a tumor suppressor depending on the cellular context and tumor stage (53). TGF- $\beta$ signaling pathways have been reported to be associated with epithelial-mesenchymal transition, breast cancer stem cell maintenance and breast cancer metastasis $(54,55)$. Additionally, estrogen and ErbB signaling pathways are associated with breast carcinogenesis, invasion and cell growth (56). Notably, agents co-targeting the estrogen and ErbB signaling pathways have been used in the clinic and demonstrated a benefit for breast cancer patients (56). The results of the current study suggest that miR-3646, -4484 and 
A Enriched pathways for miR-3646 target genes

Ubiquitin mediated proteolysis - homo sapiens (human)

Thyroid hormone signaling pathway - homo sapiens (human)

GABAergic synapse - homo sapiens (human)

Cholinergic synapse - homo sapiens (human)

Retrograde endocannabinoid signaling - homo sapiens (human)

Axon guidance - homo sapiens (human)

Rap1 signaling pathway - homo sapiens (human)

Amphetamine addiction - homo sapiens (human)

Oocyte meiosis - homo sapiens (human)

TGF- $\beta$ signaling pathway - homo sapiens (human)

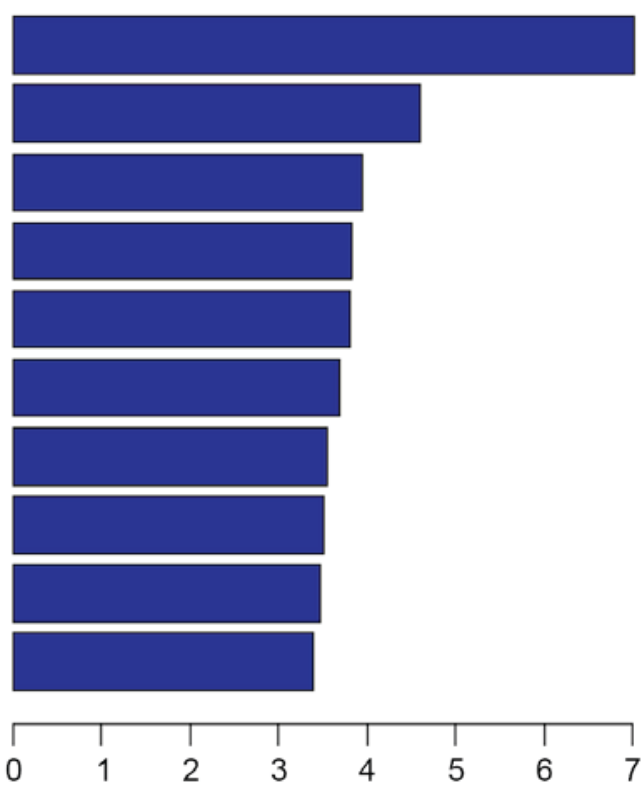

B

Enriched pathways for miR-4484 target genes

ErbB signaling pathway - homo sapiens (human)

Estrogen signaling pathway - homo sapiens (human)

Chronic myeloid leukemia - homo sapiens (human)

Bacterial invasion of epithelial cells - homo sapiens (human)

Insulin signaling pathway - homo sapiens (human)

Chemokine signaling pathway - homo sapiens (human)

Endocytosis - homo sapiens (human)

Focal adhesion - homo sapiens (human)

Cholinergic synapse - homo sapiens (human)

Adherans junction - homo sapiens (human)

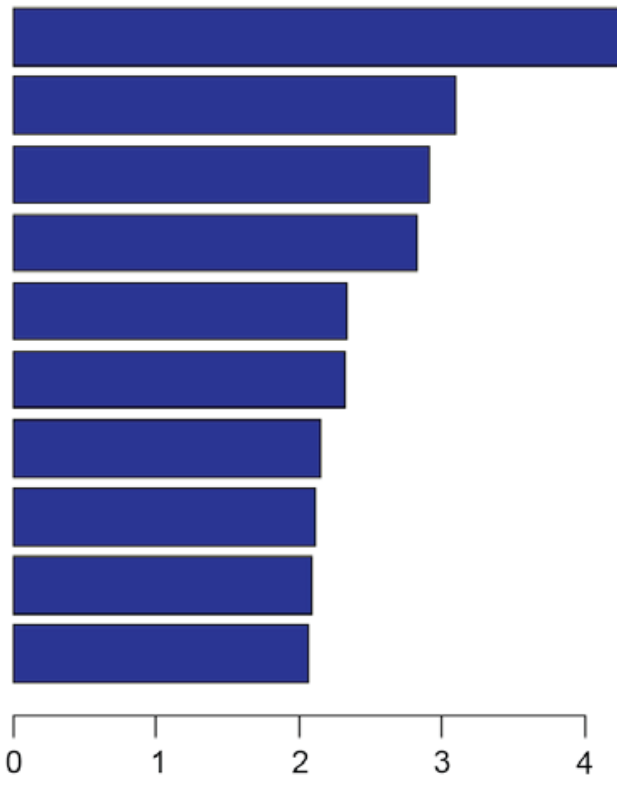

C

Enriched pathways for miR-4732-5p target genes

Proteoglycans in cancer - homo sapiens (human)

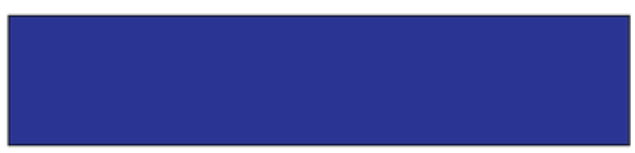

Ubiquitin mediated proteolysis - homo sapiens (human)
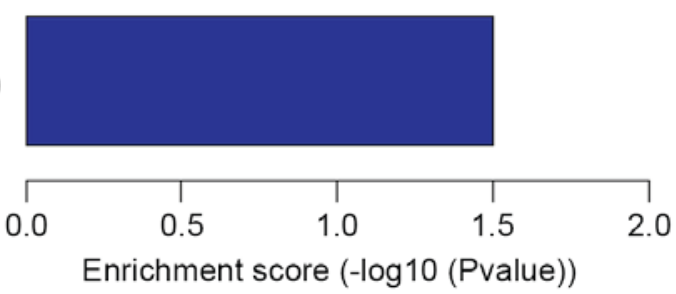

Figure 3. Kyoto Encyclopedia of Gene and Genome pathway enrichment analysis for the target genes of miR-3646, -4484 and $-4732-5 p$. The top 10 enriched pathways are shown for (A) miR-3646 and (B) miR-4484. (C) The two most significantly enriched pathways for miR-4732-5p. miR, micro RNA; GABA, $\gamma$ aminobutyric acid; TGF- $\beta$, transforming growth factor $\beta$; ErbB, epidermal growth factor receptor. 


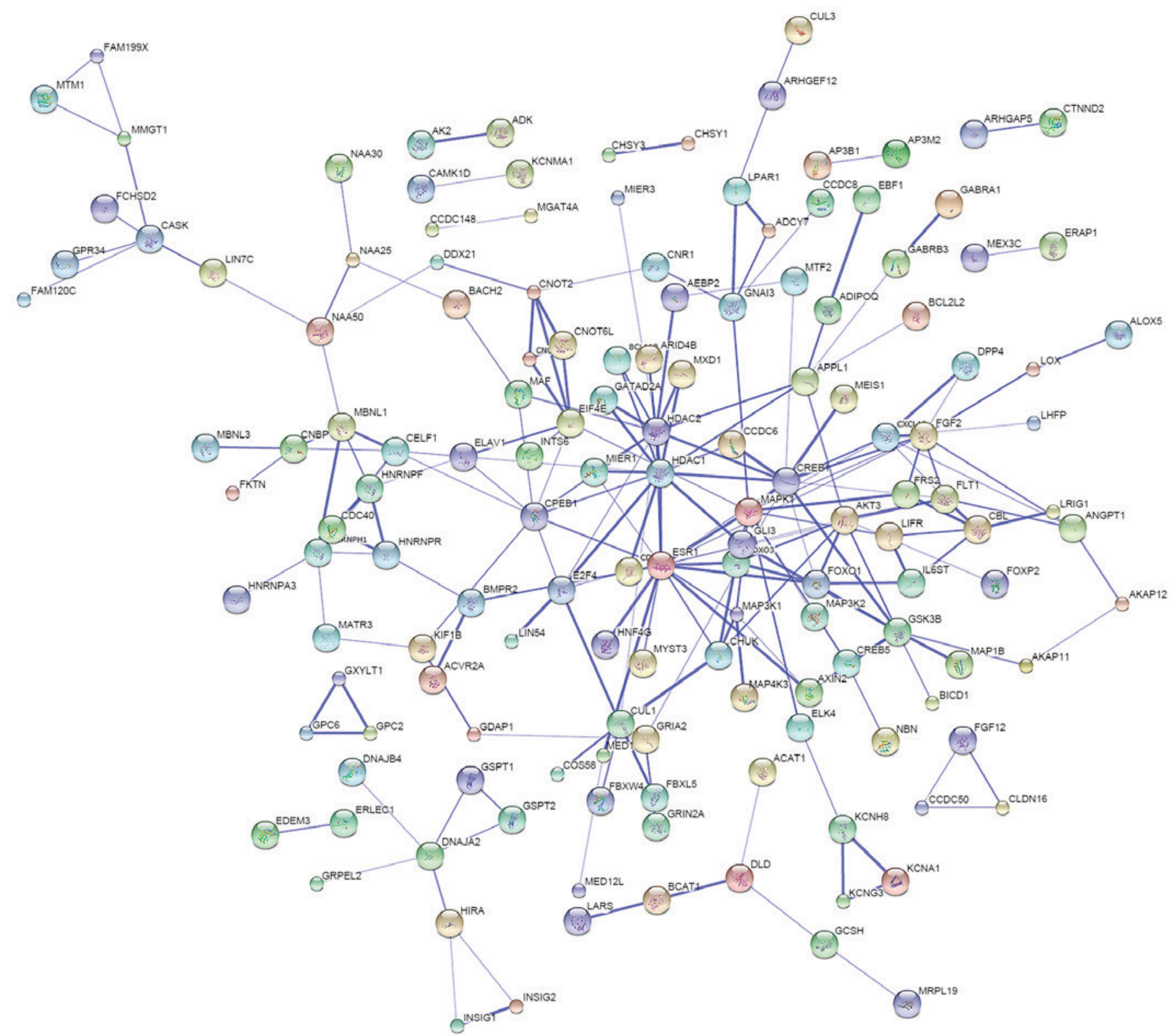

Figure 4. Protein-protein interaction analysis performed using the Search Tool for the Retrieval of Interacting Genes database revealed that the targets of miR-3646, -4484 and -4732-5p may interact with each other. The disconnected nodules are hidden. Bold lines indicate a stronger association.

-4732-5p serve important roles in human cancers, including breast cancer. However, this requires experimental validation and further investigation.

To the best of our knowledge, there are few previous studies investigating miR-3646, -4484 and -4732-5p. Rogler et al (57) reported that miR-3646 exhibited strong expression in Caucasian bladder cancer cell lines and tumor tissues. Only one report identified a clinical significance of miR-4484 in human cancer; high serum expression of miR-4484 was found to predict lymph node metastasis in patients with early-stage cervical squamous cell carcinoma (58). Pouladi et al (59) predicted a binding site for miR-4732-5p in the 5' UTR of telomerase cajal body protein 1 mRNA. In addition, miR-4732-5p has been revealed to be closely associated with recurrence in gastric cancer patients treated with S-1 adjuvant chemotherapy (60). Importantly, the TF-mRNA regulatory network constructed in the present study revealed that the target genes of the miRNAs investigated were potentially regulated by numerous transcription factors, which highlights the critical roles of these miRNAs and their targets in physiological and pathological processes. Given the uncharacterized roles and underlying molecular mechanisms of miR-3646, -4484 and -4732-5p in human cancer, additional studies are required to fully explore their functions. The results of the bioinformatics analysis performed in the present study provide a theoretical basis for further research.

In conclusion, the present study identified that miR-3646, -4484 and $-4732-5 p$ are associated with breast cancer development and progression, and in particular with certain distinct signaling pathways within these processes. The target genes of miR-3646, -4484 and $-4732-5 p$ were predicted in the current study, with the findings indicating that miR-3646, -4484 and $-4732-5 p$ are associated with 

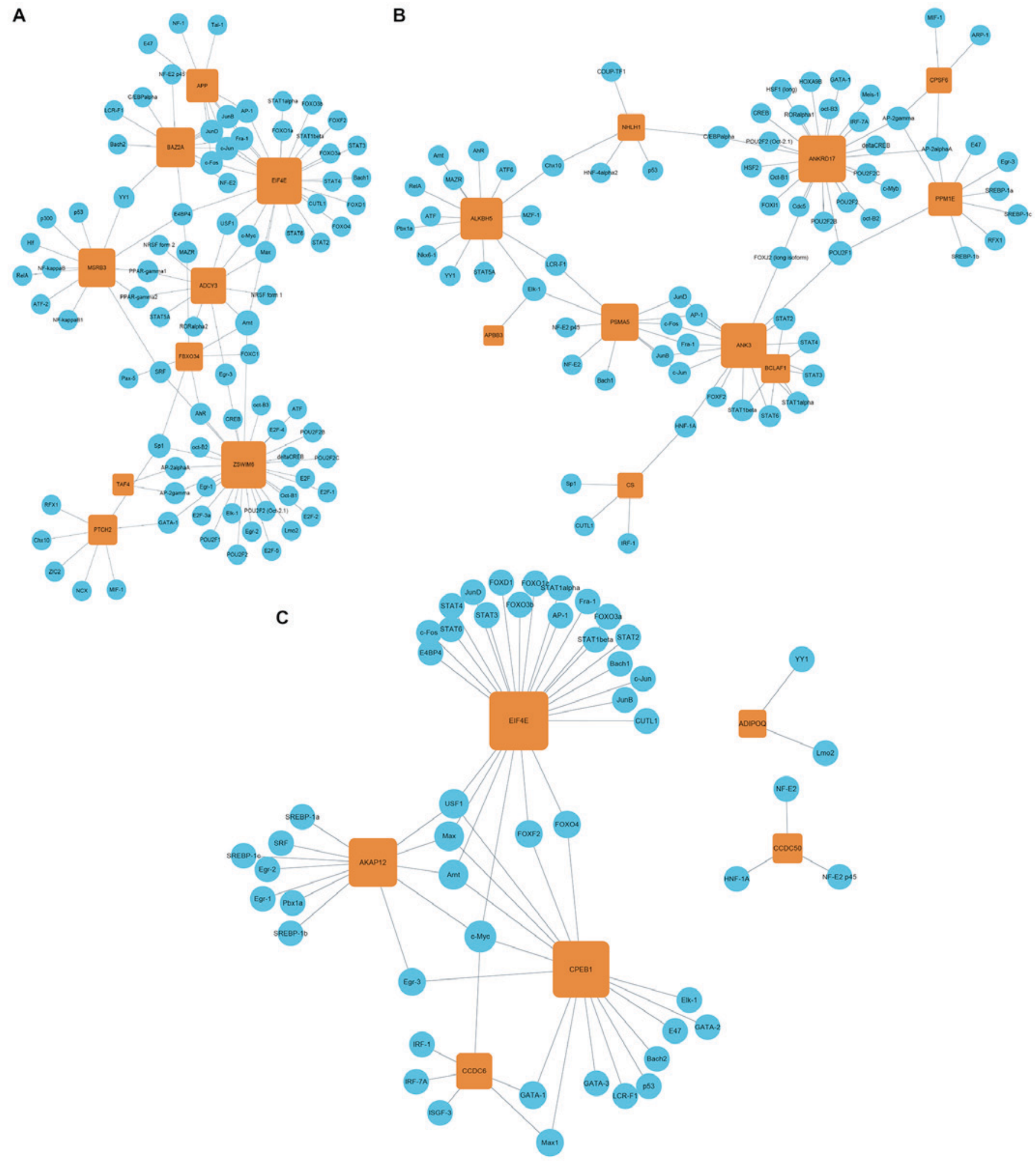

Figure 5. TF-mRNA network construction for (A) miR-4484, (B) miR-4732-5p and (C) miR-3646. Blue circles represent TFs; and orange squares represent the downstream genes regulated by corresponding TFs; lines between TFs and downstream genes represent a regulatory relationship; the size of the squares represents the number of TFs that may regulate the corresponding gene. TF, transcription factor; miR, microRNA.

signaling pathways important in cancer, including breast carcinogenesis and progression. The current study provides a comprehensive view of the functions and molecular mechanisms of miR-3646, -4484 and $-4732-5 p$ from a bioinformatics perspective, and will aid in future experimental studies.

\section{Acknowledgements}

The present study was supported by the Shandong Key Research and Development Plan (grant no. 2016GSF201128). The authors thank Cloud-Seq Company (Shanghai, China) for the technical assistance in bioinformatic analysis. 


\section{References}

1. Siegel RL, Miller KD and Jemal A: Cancer statistics, 2016. CA Cancer J Clin 66: 7-30, 2016.

2. Gülay H, Bora S, Kilicturgay S, Hamaloglu E and Göksel HA: Management of nipple discharge. J Am Coll Surg 178: 471-474, 1994.

3. Vargas HI, Vargas MP, Eldrageely K, Gonzalez KD and Khalkhali I: Outcomes of clinical and surgical assessment of women with pathological nipple discharge. Am Surg 72: 124-128, 2006.

4. Jardines L: Management of nipple discharge. Am Surg 62: 119-122, 1996.

5. Murad TM, Contesso G and Mouriesse H: Nipple discharge from the breast. Ann Surg 195: 259-264, 1982.

6. Sabel MS, Helvie MA, Breslin T, Curry A, Diehl KM, Cimmino VM, Chang AE and Newman LA: Is duct excision still necessary for all cases of suspicious nipple discharge? Breast J 18: 157-162, 2012.

7. Kapenhas-Valdes E, Feldman SM and Boolbol SK: The role of mammary ductoscopy in breast cancer: A review of the literature. Ann Surg Oncol 15: 3350-3360, 2008.

8. López-Ríos F, Vargas-Castrillón J, González-Palacios F and de Agustín PP: Breast carcinoma in situ in a male. Report of a case diagnosed by nipple discharge cytology. Acta cytol 42 : 742-744, 1998

9. Calin GA and Croce CM: MicroRNA signatures in human cancers. Nat Rev Cancer 6: 857-866, 2006.

10. Tsukamoto Y, Nakada C, Noguchi T, Tanigawa M, Nguyen LT, Uchida T, Hijiya N, Matsuura K, Fujioka T, Seto M and Moriyama M: MicroRNA-375 is downregulated in gastric carcinomas and regulates cell survival by targeting PDK1 and 14-3-3zeta. Cancer Res 70: 2339-2349, 2010.

11. Wang YW, Shi DB, Chen X, Gao C and Gao P: Clinicopathological significance of microRNA-214 in gastric cancer and its effect on cell biological behaviour. PloS one 9: e91307, 2014.

12. Fabbri M: miRNAs as molecular biomarkers of cancer. Expert Rev Mol Diagn 10: 435-444, 2010.

13. Zhang K, Zhang Y, Liu C, Xiong Y and Zhang J: MicroRNAs in the diagnosis and prognosis of breast cancer and their therapeutic potential (Review). Int J Oncol 45: 950-958, 2014.

14. Zhong Z, Dong Z, Yang L, Chen X and Gong Z: Inhibition of proliferation of human lung cancer cells by green tea catechins is mediated by upregulation of let-7. Exp Ther Med 4: 267-272, 2012.

15. Takamizawa J, Konishi H, Yanagisawa K, Tomida S, Osada H, Endoh H, Harano T, Yatabe Y, Nagino M, Nimura Y, et al: Reduced expression of the let-7 microRNAs in human lung cancers in association with shortened postoperative survival Cancer Res 64: 3753-3756, 2004.

16. Stahlhut $C$ and Slack FJ: Combinatorial ction of microRNAs let-7 and miR-34 effectively synergizes with erlotinib to suppress non-small cell lung cancer cell proliferation. Cell Cycle 14: 2171-2180, 2015.

17. Cui L, Zhang X, Ye G, Zheng T, Song H, Deng H, Xiao B, Xia T, $\mathrm{Yu}$ X, Le Y and Guo J: Gastric juice microRNAs as potential biomarkers for the screening of gastric cancer. Cancer 119: 1618-1626, 2013.

18. Zhang K, Zhao S, Wang Q, Yang HS, Zhu J and Ma R: Identification of microRNAs in nipple discharge as potential diagnostic biomarkers for breast cancer. Ann Surg Oncol 22 (Suppl 3): S536-S544, 2015.

19. Wong $\mathrm{N}$ and Wang $\mathrm{X}$ : miRDB: An online resource for microRNA target prediction and functional annotations. Nucleic Acids Res 43: D146-D152, 2015.

20. Gene Ontology Consortium, Blake JA, Dolan M, Drabkin H, Hill P, Li N, Sitnikov D, Bridges S, Burgess S, Buza T, et al: Gene ontology annotations and resources. Nucleic Acids Res 41: D530-D535, 2013.

21. Kanehisa M, Goto S, Sato Y, Furumichi M and Tanabe M: KEGG for integration and interpretation of large-scale molecular data sets. Nucleic Acids Res 40: D109-D114, 2012.

22. Franceschini A, Szklarczyk D, Frankild S, Kuhn M, Simonovic M, Roth A, Lin J, Minguez P, Bork P, von Mering C and Jensen LJ: STRING v9.1: Protein-protein interaction networks, with increased coverage and integration. Nucleic Acids Res 41: D808-D815, 2013.

23. Vargas HI, Romero L and Chlebowski RT: Management of bloody nipple discharge. Curr Treat Options Oncol 3: 157-161, 2002 .
24. Dinkel HP, Trusen A, Gassel AM, Rominger M, Lourens S, Müller T and Tschammler A: Predictive value of galactographic patterns for benign and malignant neoplasms of the breast in patients with nipple discharge. Br J Radiol 73: 706-714, 2000.

25. Sauter ER, Ehya H, Babb J, Diamandis E, Daly M, Klein-Szanto A, Sigurdson E, Hoffman J, Malick J and Engstrom PF: Biological markers of risk in nipple aspirate fluid are associated with residual cancer and tumour size. Br J Cancer 81: 1222-1227, 1999.

26. Zhang K, Zhao S, Wang Q, Yang HS, Zhu J and Ma R: Identification of microRNAs in nipple discharge as potential diagnostic biomarkers for breast cancer. Ann Surg Oncol 22 Suppl 3: S536-S544, 2015.

27. Liu Y, Zhou J, Zhang C, Fu W, Xiao X, Ruan S, Zhang Y, Luo X and Tang M: HLJ1 is a novel biomarker for colorectal carcinoma progression and overall patient survival. Int J Clin Exp Pathol 7: 969-977, 2014.

28. Simões-Correia J, Silva DI, Melo S, Figueiredo J, Caldeira J, Pinto MT, Girão H, Pereira P and Seruca R: DNAJB4 molecular chaperone distinguishes WT from mutant E-cadherin, determining their fate in vitro and in vivo. Hum Mol Genet 23: 2094-2105, 2014.

29. Chen HW, Lee JY, Huang JY, Wang CC, Chen WJ, Su SF, Huang CW, Ho CC, Chen JJ, Tsai MF, et al: Curcumin inhibits lung cancer cell invasion and metastasis through the tumor suppressor HLJ1. Cancer Res 68: 7428-7438, 2008.

30. Tsai MF, Wang CC, Chang GC, Chen CY, Chen HY, Cheng CL, Yang YP, Wu CY, Shih FY, Liu CC, et al: A new tumor suppressor DnaJ-like heat shock protein, HLJ1 and survival of patients with non-small-cell lung carcinoma. J Natl Cancer Inst 98: 825-838, 2006.

31. Mantzoros C, Petridou E, Dessypris N, Chavelas C, Dalamaga M, Alexe DM, Papadiamantis Y, Markopoulos C, Spanos E, Chrousos G and Trichopoulos D: Adiponectin and breast cancer risk. J Clin Endocrinol Metab 89: 1102-1107, 2004.

32. Falk Libby E, Liu J, Li YI, Lewis MJ, Demark-Wahnefried W and Hurst DR: Globular adiponectin enhances invasion in human breast cancer cells. Oncol Lett 11: 633-641, 2016.

33. Chen L, Ye C, Huang Z, Li X, Yao G, Liu M, Hu X, Dong J and Guo Z: Differentially expressed genes and potential signaling pathway in Asian people with breast cancer by preliminary analysis of a large sample of the microarray data. Nan Fang Yi Ke Da Xue Xue Bao 34: 807-812, 2014 (In Chinese).

34. Nairismägi ML, Vislovukh A, Meng Q, Kratassiouk G, Beldiman C, Petretich M, Groisman R, Füchtbauer EM, Harel-Bellan A and Groisman I: Translational control of TWIST1 expression in MCF-10A cell lines recapitulating breast cancer progression. Oncogene 31: 4960-4966, 2012.

35. Hansen CN, Ketabi Z, Rosenstierne MW, Palle C, Boesen HC and Norrild B: Expression of CPEB, GAPDH and U6snRNA in cervical and ovarian tissue during cancer development. APMIS 117: 53-59, 2009.

36. Caldeira J, Simões-Correia J, Paredes J, Pinto MT, Sousa S, Corso G, Marrelli D, Roviello F, Pereira PS, Weil D, et al: CPEB1, a novel gene silenced in gastric cancer: A Drosophila approach. Gut 61: 1115-1123, 2012.

37. Xiaoping L, Zhibin Y, Wenjuan L, Zeyou W, Gang X, Zhaohui L, Ying Z, Minghua W and Guiyuan L: CPEB1, a histone-modified hypomethylated gene, is regulated by miR-101 and involved in cell senescence in glioma. Cell Death Dis 4: e675, 2013.

38. Kochanek DM and Wells DG: CPEB1 regulates the expression of MTDH/AEG-1 and glioblastoma cell migration. Mol Cancer Res 11: 149-160, 2013

39. Miyazaki T, Ikeda K, Horie-Inoue K and Inoue S: Amyloid precursor protein regulates migration and metalloproteinase gene expression in prostate cancer cells. Biochem Biophys Res Commun 452: 828-833, 2014.

40. Yoshitomi T, Kawakami K, Enokida H, Chiyomaru T, Kagara I, Tatarano S, Yoshino H, Arimura H, Nishiyama K, Seki N and Nakagawa M: Restoration of miR-517a expression induces cell apoptosis in bladder cancer cell lines. Oncol Rep 25: 1661-1668, 2011.

41. Chen Y, Wang Y, Song H, Wang J, Yang H, Xia Y, Xue J, Li S, Chen $\mathrm{M}$ and $\mathrm{Lu} \mathrm{Y}$ : Expression profile of apoptosis-related genes potentially explains early recurrence after definitive chemoradiation in esophageal squamous cell carcinoma. Tumour Biol 35: 4339-4346, 2014.

42. Carroll M and Borden KL: The oncogene eIF4E: using biochemical insights to target cancer. J Interferon Cytokine Res 33: 227-238, 2013. 
43. Pettersson F, Del Rincon SV, Emond A, Huor B, Ngan E, $\mathrm{Ng}$ J, Dobocan MC, Siegel PM and Miller WH Jr: Genetic and Pharmacologic inhibition of eIF4E reduces breast cancer cell migration, invasion and metastasis. Cancer Res 75: 1102-1112, 2015.

44. Hu A, Sun M, Yan D and Chen K: Clinical significance of mTOR and eIF4E expression in invasive ductal carcinoma. Tumori 100: 541-546, 2014

45. Yin X, Kim RH, Sun G, Miller JK and Li BD: Overexpression of eukaryotic initiation factor $4 \mathrm{E}$ is correlated with increased risk for systemic dissemination in node-positive breast cancer patients. J Am Coll Surg 218: 663-671, 2014.

46. Spanjaard E, Smal I, Angelopoulos N, Verlaan I, Matov A, Meijering E, Wessels L, Bos H and de Rooij J: Quantitative imaging of focal adhesion dynamics and their regulation by HGF and Rap1 signaling. Exp Cell Res 330: 382-397, 2015.

47. Che YL, Luo SJ, Li G, Cheng M, Gao YM, Li XM, Dai JM, He H, Wang J, Peng HJ, et al: The C3G/Rap1 pathway promotes secretion of MMP-2 and MMP-9 and is involved in serous ovarian cancer metastasis. Cancer lett 359: 241-249, 2015.

48. Alemayehu M, Dragan M, Pape C, Siddiqui I, Sacks DB, Di Guglielmo GM, Babwah AV and Bhattacharya M: $\beta$-Arrestin2 regulates lysophosphatidic acid-induced uman breast tumor cell migration and invasion via Rap1 and IQGAP1. PloS one 8: e56174,2013.

49. Ahmed SM, Thériault BL, Uppalapati M, Chiu CW, Gallie BL, Sidhu SS and Angers S: KIF14 negatively regulates Rapla-Radil signaling during breast cancer progression. J Cell Biol 199: 951-967, 2012

50. McSherry EA, Brennan K, Hudson L, Hill AD and Hopkins AM: Breast cancer cell migration is regulated through junctional adhesion molecule-A-mediated activation of Rap1 GTPase. Breast Cancer Res 13: R31, 2011.
51. Itoh M, Nelson CM, Myers CA and Bissell MJ: Rap1 integrates tissue polarity, lumen formation, and tumorigenic potential in human breast epithelial cells. Cancer Res 67: 4759-4766, 2007.

52. Zhao B and Chen YG: Regulation of TGF- $\beta$ Signal Transduction. Scientifica (Cairo) 2014: 874065, 2014.

53. Zarzynska JM: Two faces of TGF-betal in breast cancer. Mediators Inflamm 2014: 141747, 2014.

54. Kotiyal S and Bhattacharya S: Breast cancer stem cells, EMT and therapeutic targets. Biochem Biophys Res Commun 453: 112-116, 2014.

55. Imamura T, Hikita A and Inoue Y: The roles of TGF- $\beta$ signaling in carcinogenesis and breast cancer metastasis. Breast Cancer 19: 118-124, 2012.

56. Mehta A and Tripathy D: Co-targeting estrogen receptor and HER2 pathways in breast cancer. Breast 23: 2-9, 2014.

57. Rogler A, Hoja S, Socher E, Nolte E, Wach S, Wieland W, Hofstädter F, Goebell PJ, Wullich B, Hartmann A and Stoehr R: Role of two single nucleotide polymorphisms in secreted frizzled related protein 1 and bladder cancer risk. Int J Clin Exp Pathol 6: 1984-1998, 2013

58. Chen J, Yao D, Li Y, Chen H, He C, Ding N, Lu Y, Ou T, Zhao S, Li L and Long F: Serum microRNA expression levels can predict lymph node metastasis in patients with early-stage cervical squamous cell carcinoma. Int J Mol Med 32: 557-567, 2013.

59. Pouladi N, Kouhsari SM, Feizi MH, Gavgani RR and Azarfam P: Overlapping region of p53/wrap53 transcripts: Mutational analysis and sequence similarity with microRNA-4732-5p. Asian Pac J Cancer Prev 14: 3503-3507, 2013.

60. Omura T, Shimada Y, Nagata T, Okumura T, Fukuoka J, Yamagishi F, Tajika S, Nakajima S, Kawabe A and Tsukada K: Relapse-associated microRNA in gastric cancer patients after S-1 adjuvant chemotherapy. Oncol Rep 31: 613-618, 2014. 